In der Rubrik „Literatur kompakt" werden die wichtigsten Originalarbeiten aus der internationalen Fachliteratur referiert.

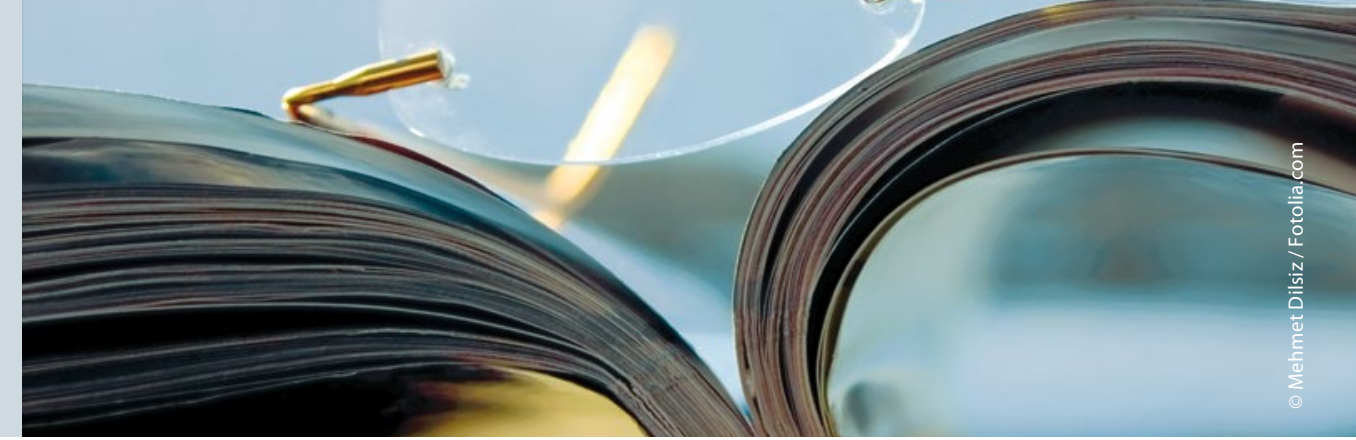

\section{Probiotika zur Allergie-Primärprävention: schwache Evidenz}

\begin{abstract}
Probiotika modulieren die Darmimmunologie. Doch sollen sie auch systemische Immuneffekte entfalten und werden deshalb unter anderem für die Primärprävention von Allergien empfohlen. Die World Allergy Organization fand für diese Empfehlungen aber nur eine schwache Evidenz.
\end{abstract}

P robiotika sind lebensfähige Mikroorganismen, meist Bifido- oder Laktobakterien-Spezies, die eingearbeitet in Lebensmitteln wie Milch oder Jogurt oder als pharmazeutische Präparate an-

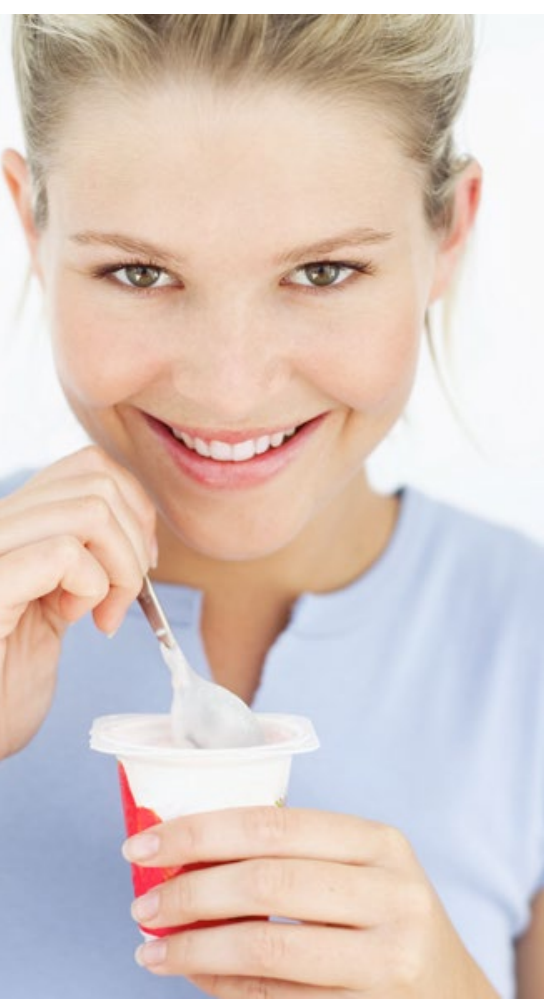

Vergebliche Mühe? geboten werden. Ihre immunmodulatorischen, gesundheitsfördernden und auch allergiepräventiven Eigenschaften werden besonders in Laienmedien intensiv beworben. Um dem Arzt eine evidenzbasierte Beratung zur möglichen Allergieprävention zu ermöglichen, veröffentlichte die World Allergy Organization (WAO) jetzt eine aktuelle Leitlinie, basierend auf publizierten kontrollierten Studien zu diesem Thema.

Insgesamt war die Evidenz zu primärpräventiven Effekten nur schwach. Trotzdem gab die WAO einige Empfehlungen, die aufgrund des niedrigen Evidenzniveaus aber mit Vorsicht interpretiert werden sollten. Das heißt, in einigen Fällen kann sich ein präventiver Effekt einstellen, bei der Mehrheit wohl eher nicht. Nebenwirkungen sind allerdings in der Regel nicht zu erwarten, sodass kein Schaden durch die Empfehlung entsteht. In der ärztlichen Beratung orientiert sich eine mögliche ProbiotikaEmpfehlung aufgrund der schwachen Evidenz daher auch am ehesten am Leidensdruck sowie den Werten und Einstelllungen der Eltern.

_Empfehlung 1: Probiotika können schwangeren Frauen angeboten werden, die ein Hochrisikokind erwarten. Zumindest die Ekzemrate reduziert sich durch diese Maßnahme leicht.
Basis für diese Empfehlung waren acht systematische Reviews, der postnatale Follow-up-Zeitraum lag zwischen zwei und 24 Monaten.

-Empfehlung 2: Probiotika können stillenden Müttern von Hochrisikokindern angeboten werden. Auch durch diese Maßnahme können Ekzeme verhindert werden, andere allergische Erkrankungen aber nicht. Die 13 zu dieser Fragestellung gefundenen kontrollierten Studien dauerten ebenfalls zwischen zwei und 24 Monaten.

_Empfehlung 3: Eine Probiotika-Supplementation kann bei Hochrisikokindern primärpräventive Effekte auf die Ekzementwicklung entfalten. Diese Empfehlung leitet sich aus insgesamt fünf systematischen Reviews ab, wobei sich die meisten Studien auf die Entwicklung eines Ekzems konzentrierten. Die Follow-up-Zeiten lagen zwischen vier und 36 Monaten. Bei anderen untersuchten Allergien wie Nahrungsmittelallergien oder allergische Rhinitis wurden keine Schutzeffekte festgestellt.

Fazit: Die Evidenz einer primärpräventiven antiallergischen Wirkung von Probiotika ist insgesamt dürftig. Hinweise ergeben sich für einen Nutzen in der Ekzem-Prävention bei Hochrisikokindern. Eine Probiotika-Supplementation für Schwangere, Stillende und die Kinder kann deshalb im Bedarfsfall und mit der gebotenen Zurückhaltung empfohlen werden.

Dr. Barbara Kreutzkamp

Fiocchi A et al. World Allergy OrganizationMcMaster University. Guidelines for allergic disease prevention (GLAD-P): Probiotics. World Allergy Organ J 2015; 8: 4 